\title{
Fixed Echo Rejection in Sodar Using Noncoherent Matched Filter Detection and Gaussian Mixture Model-Based Postprocessing
}

\author{
PAUl KENDRICK AND SABINE VON HÜNERBEIN \\ Acoustics Research Centre, University of Salford, Manchester, United Kingdom
}

(Manuscript received 7 June 2018, in final form 10 October 2018)

\begin{abstract}
Doppler sodar is a technology used for acoustic-based remote sensing of the lower planetary boundary layer. Sodars are often used to measure wind profiles; however, they suffer from problems caused by noise (both acoustic and electrical) and echoes from fixed objects, which can bias radial velocity estimates. An experimental bistatic sodar was developed with 64 independent channels. The device enables flexible beamforming; beams can be tilted at the same angle irrelevant of frequency, a limitation in most commercial devices. This paper presents an alternative sodar signal-processing algorithm for wind profiling using a multifrequency stepped-chirp pulse. A noncoherent matched filter was used to analyze returned signals. The noncoherent matched filter combines radial velocity estimates from multiple frequencies into a single optimization. To identify and separate sources of backscatter, noise, and fixed echoes, a stochastic patternrecognition technique, Gaussian mixture modeling, was used to postprocess the noncoherent matched filter data. This method allowed the identification and separation of different stochastic processes. After identification, noise and fixed echo components were removed and a clean wind profile was produced. This technique was compared with traditional spectrum-based radial velocity estimation methods, and an improvement in the rejection of fixed echo components was demonstrated; this is one of the major limitations of sodar performance when located in complex terrain and urban environments.
\end{abstract}

\section{Introduction}

Sodar operates by transmitting pulses of sound into the atmosphere and analyzing the sound backscattered from moving turbulent fluctuations. Sodars have a variety of applications, including wind velocity profiling (Peters et al. 1984) and measurements of turbulence and stability parameters (Petenko et al. 2014). However, backscattered sound may originate from both atmospheric turbulence and stationary objects such as buildings or masts. Because of the zero-Doppler shift on these echoes, in wind velocity profiling this can bias estimates. Fixed echoes from sidelobes of the transmitted beam pattern are one of the main sources of error in sodar measurements (Bradley and von Hünerbein 2007). Fixed echoes are usually minimized by placing the sodar transponder away from tall objects such as trees, masts, buildings, and slopes with large stone boulders (AQSystem 2013). Most sodars use acoustic baffles to reduce sidelobes and thus control fixed echoes. Sodar manufacturers provide guidance as to how to reduce the risk from fixed

Corresponding author: Paul Kendrick, p.kendrick@salford.ac.uk echoes. AQSystem (2013) suggests plotting complete (no data rejection) wind speed profiles on a log-log scale. Should the relationship be nonlinear, this indicates deviation from the expected power-law relationship and could indicate the presence of fixed echoes. Similarly, fixed echoes may be detected as discontinuities in radial velocity profiles of amplitude or frequency (Kalogiros and Helmis 1999). Kalogiros and Helmis (1999) also proposed a method utilizing the wavelet transform to better locate fixed echoes in time. The variability of the radial wind velocity over a scattering volume causes spectral broadening of the returned signal (Mayer 2005). Spectral broadening is also linked to the beamwidth of the device (Quintarelli and Bergstrom 2001). As such, commercial sodars may also use the width of Doppler spectrum peaks to indicate the presence of fixed echoes (Antoniou et al. 2003). However, such methods are sensitive to the chosen threshold and may lead to poor data availability. Alternatively, a bistatic sodar can be used where the Doppler shift in the backscattered signal is greater than an equivalent monostatic implementation (S. Bradley et al. 2012); this makes the differentiation between fixed echo and turbulent backscatter easier. 
This paper aims to address some of the issues associated with fixed echoes. A flexible bistatic sodar was developed with two, 32-channel, two-dimensional transducer arrays. Each transducer had its own independent signal path to allow more control over beamforming behavior than other sodars. Backscattered return signals were analyzed using a noncoherent matched filter, which combined the estimation of Doppler shifts from multiple sequential pulses of different frequencies into a single optimization. Gaussian mixture models (GMM) were then used to analyze received signals; this processing method shows interesting advantages in fixed echo detection and rejection, one of the main limitations in sodar performance.

\section{Background and method}

\section{a. A flexible sodar design}

Sodars may be implemented using horn antenna with parabolic dishes for capturing backscatter (Argentini et al. 2013) but are also often implemented as twodimensional transducer arrays where beam steering is achieved by introducing phase shifts between groups of transducers. Bradley and von Hünerbein (2007) compare several sodar technologies. Introducing phase shifts between groups rather than individual transducers minimizes the required number of channels; however, it also means that tilt angle is dependent on frequency, and, as such, different frequencies will backscatter from entirely different volumes of atmosphere. An experimental bistatic sodar was designed with 32 separate transducers for both receiver and transmitter, where each had an independent signal path (Fig. 1). This allows for flexible signal-processing approaches in which tilt and angle and frequency can be decoupled. A 32-channel digital-to-analog converter (DAC) and a 32-channel analog-to-digital converter (ADC) (RME M-32AD and RME M-32DA) were used, with playback and capture carried out in the MATLAB software package. The sampling frequency was $44100 \mathrm{~Hz}$. The design was bistatic, although the receiver and transmitter were closely located so that the sodar could be operated as a monostatic device. This means that there was no requirement for a switch between transmission and receive modes, and therefore the blind range was minimized. Both transmitter and receiver used 32 identical transducers (Motorola, Inc., KSN-1005A superhorn piezoelectric tweeters). More details of the implementation can be found in Kendrick et al. (2010).

Beamforming was implemented over the transmitter and receiver by applying independent time delays to each channel. A five-beam configuration was used that includes north, south, east, west, and vertical with a tilt of $20^{\circ}$. Beamforming was trivial to implement on the

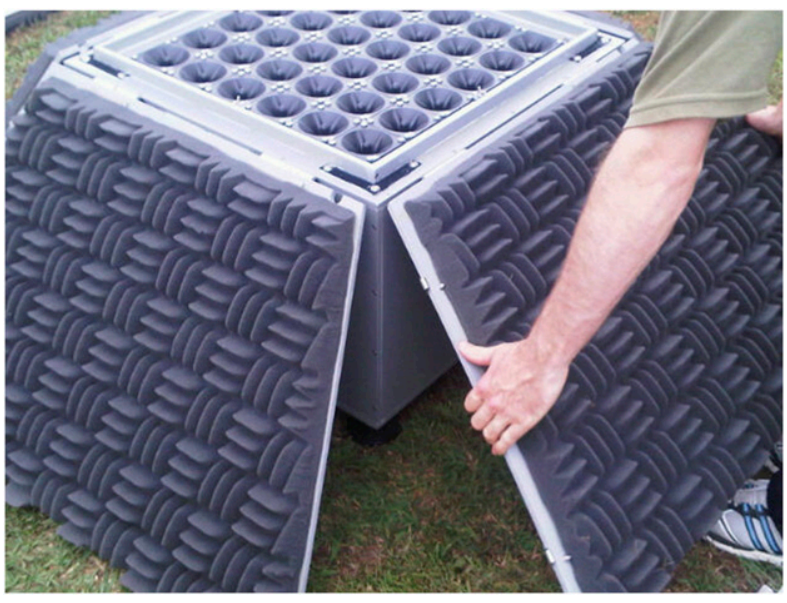

FIG. 1. Antenna array containing Motorola KSN-1005A superhorn piezoelectric tweeters with hinged baffle slabs.

transmitter, because individual delays could be applied to the pulse-generating function for each speaker. For the receiver, delays were applied to each signal by adding a linear phase term to the Fourier transform of the received signal. This was converted back into the time domain by using an inverse Fourier transform, prior to summation over all channels.

\section{b. Pulse design and matched filtering}

\section{1) STEPPED CHIRP}

The flexible nature of the sodar design ensured the different frequencies could be steered in the same directions for both transmitter and receiver. Multifrequency pulses can offer advantages in terms of flexibility and increased range in sodar (Rao et al. 2009). Therefore, a multifrequency approach was adopted, where a train of subpulses of different frequencies was generated and transmitted. This is referred to as a stepped chirp, which is a form of frequency modulation known as frequency-shift keying (FSK). The stepped chirp is a train of $M$ single-frequency pulses, each with frequency $f_{m}$, and modulated by a Gaussian window,

$$
x(t)=\sum_{m=0}^{M-1} \sin \left[2 \pi f_{m}(t-\mathrm{m} T)\right] G(t),
$$

where $m$ is the pulse number, $T$ is the pulse length, and $G(t)$ is a Gaussian window function,

$$
G(t)=\exp \left\{\left[t-\left(m T+\frac{T}{2}\right)\right]^{2} / \sigma^{2}\right\},
$$

where here $\sigma=T / 4$. The stepped chirp can be processed in multiple ways. It can be processed using a common 
approach adopted by most commercial sodars; the return signal is windowed into range gates, and the Doppler power spectrum is computed for each frequency separately. Doppler shifts and thus radial velocities are estimated by locating peaks in the spectrum. A range offset is applied to account for the delay in subpulse transmission times for different frequencies. The stepped chirp can also be analyzed using a technique known as matched filtering, which has been shown to improve performance in phased-array radar systems for weather radar (Alberts and Chilson 2011).

\section{2) MATCHED FILTER RECEIVER}

Matched filters are associated with pulse compression, a technique used in radar to increase maximum range without compromising range resolution for moving point targets (Klauder et al. 1960). Longer waveforms are transmitted; this increases the transmitted power but unlike the simple pulse, the transmitted bandwidth is also increased. This enables echoes to be unwrapped; pulse compression allows an increase in range while preserving range resolution. Bandwidth extension in pulse compression is usually achieved by frequency or phase modulation (FSK in this case). Detection involves a matched filter. A matched filter is commonly used to detect scattered signals in both point target and weather radar. Formally, a matched filter is the optimal linear filter, for a signal, for maximizing the signal-to-noise ratio within the presence of noise. It is defined as the correlation of the received signal with a local copy of the transmitted waveform,

$$
\operatorname{mf}(t)=\int_{-\infty}^{\infty} y(\tau) x^{*}(\tau-t) d \tau
$$

where the matched filter magnitude $\operatorname{mf}(t)$ at time $t$ represents the magnitude of the returned signal at a range of $c t$ meters ( $c$ is the speed of sound), $x(t)$ is the local copy of the transmitted signal, and $y(t)$ is the received signal. Bradley (1999) suggested a number of waveforms that may be appropriate for pulse compression in sodar.

Unfortunately, pulse compression for wind profiling is not possible in sodar as shown by Hargreaves et al. (2014). This is because Bragg scattering, the dominant scattering mechanism, does not provide coherent return signals. Scattering is dominated by randomly located turbulent eddies spaced by integer multiples of the transmission wavelength. Hence, differently located eddies will dominate at different wavelengths. This causes the phase response of a scattering volume to be both stochastic and nonlinear; there is not a predictable relationship between the phases of each subpulse. This breakdown of the intersubpulse phase relationship means that the advantages of pulse compression are not realized. However, a matched filter is still capable of noncoherent signal detection (Guimarães and de Souza 2015). Noncoherent matched filtering does not increase range or Doppler resolution when compared with the spectral estimation method, but, as will be demonstrated, it helpfully combines the Doppler estimation from many discrete frequencies into one optimization problem.

\section{3) DOPPLER ESTIMATION FROM MATCHED FILTERS}

When an object is moving, there is now a mismatch because of the Doppler shift between the scattered signal and the stored waveform. This mismatch results in a decrease in the matched filter output magnitude as the correlation between returned and transmitted signal is reduced. If the stored waveform can be modified to better represent the scattered signal from the moving object, then the mismatch is reduced. Therefore, it is common to use a bank of matched filters, with each representing a different radial velocity. The filter with the largest output, at a time lag (range), represents the likely radial velocity for an object at that range.

Radar matched filter banks such as those demonstrated by Othman et al. (2017) utilize a narrowband model of Doppler shift. This assumes that the wave speed is significantly greater than the target speed and as a result Doppler can be modeled as a simple shift in frequency. In sodar, wave speed and wind velocity are more similar in their orders of magnitude; thus, a wideband model of Doppler shift was employed. In this case both the shift in frequency and elongation (or compression) in time of the waveform were modeled. The degree to which the waveform is stretched is captured in a Doppler stretch parameter $\alpha$ that has a monotonic relationship with the radial velocity $v$ as follows:

$$
\alpha(v)=\frac{c-v}{c+v} .
$$

A delayed, stretched version of the stepped chirp, representing an echo from target at $v$, can be expressed as

$$
x(t, v)=\sum_{m=1}^{M} \sin \left(2 \pi f_{m}\{\alpha(v)[t-(m-1) T]\}\right) G(t, v),
$$

where

$$
G(t, v)=\exp \left(-\{\alpha(v) t-[(m-1) T+T / 2] / \sigma\}^{2}\right) .
$$

A matched filter bank was defined that contains waveforms representing a range of target radial 


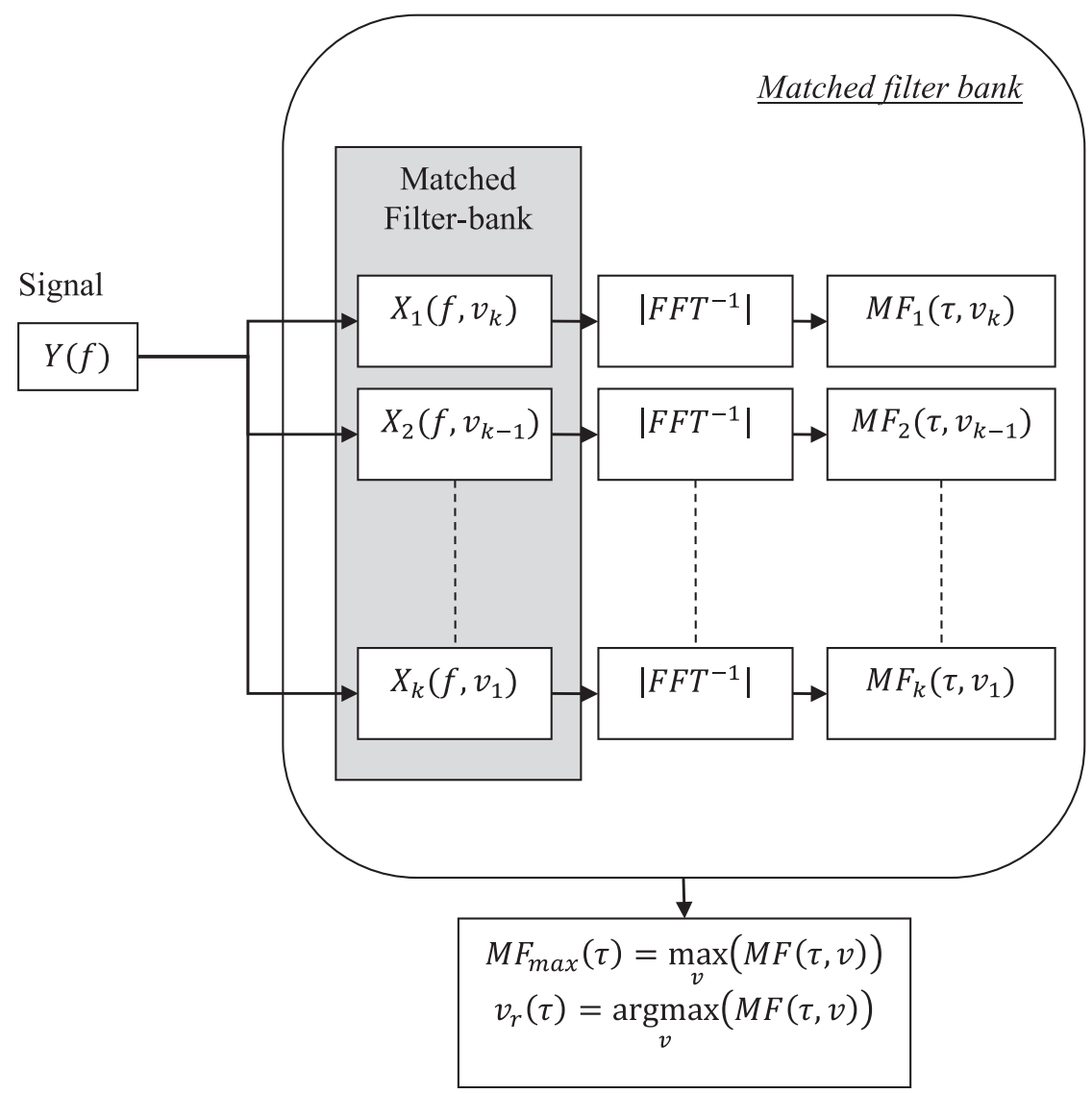

FIG. 2. System diagram for matched filter bank implementation.

velocities $v_{k}$. These waveforms were then correlated with the scattered signal $y(t)$; the matched filter output for a particular range and velocity is

$$
\operatorname{mf}_{k}\left(t, v_{k}\right)=\left|\sqrt{\alpha\left(v_{k}\right)} \int_{-\infty}^{\infty} y(\tau) x^{*}\left[\alpha\left(v_{k}\right)(\tau-t)\right] d \tau\right| .
$$

The matched filter bank was implemented as shown in Fig. 2. A grid of trial radial velocities was defined over a realistic range. With an assumption of a maximum horizontal wind velocity of $20 \mathrm{~m} \mathrm{~s}^{-1}$ and a beam tilt of $20^{\circ}$, the maximum radial velocity is about $7 \mathrm{~m} \mathrm{~s}^{-1}$; therefore, a grid of trial velocities was defined between -7 and $7 \mathrm{~m} \mathrm{~s}^{-1}$ in $0.05 \mathrm{~m} \mathrm{~s}^{-1}$ steps (resolution defined by the available RAM at a sampling frequency of $44100 \mathrm{~Hz}$ ). A matched filter was computed for each trial radial velocity, and cross-correlation calculations were carried out in the frequency domain for speed,

$$
\operatorname{mf}_{k}\left(\tau, v_{k}\right)=F^{-1}\left\{F[y(t)] F\left[x_{d}\left(t, v_{k}\right)\right]^{*}\right\},
$$

where $F$ indicates a Fourier transform and $F^{-1}$ is the inverse Fourier transform. The resulting matched filter output is downsampled from 44100 to $400 \mathrm{~Hz}$ to reduce memory requirements. This reduces the range resolution from 7.6 to $0.84 \mathrm{~m}$, although the effective resolution is still determined by the subpulse duration. The maximum value for each time lag was found,

$$
\begin{aligned}
\mathrm{mf}_{\max }(\tau) & =\max _{v_{k}}\left[\operatorname{mf}_{k_{\text {downsampled }}}\left(\tau, v_{k}\right)\right] \text { and } \\
v_{r}(\tau) & =\underset{v_{k}}{\operatorname{argmax}}\left[\operatorname{mf}_{k_{\text {downsampled }}}\left(\tau, v_{k}\right)\right] .
\end{aligned}
$$

This results in two outputs, $\operatorname{mf}_{\max }(\tau)$ and $v_{r}(\tau)$. The time lag $\tau$ represents the range $(c \tau) ; \operatorname{mf}_{\max }(\tau)$ indicates the returned signal strength for a particular range; and $v_{r}(\tau)$ is the estimated radial velocity for each lag. The signal-to-noise ratio (SNR) of the backscatter was estimated as follows:

$$
\mathrm{SNR}_{\text {matched }}(\tau)=20 \log _{10}\left[\frac{\mathrm{mf}_{\text {max }}(\tau)}{N_{\mathrm{mf}}}\right] \text {, }
$$

where the noise level $N_{\mathrm{mf}}$ is the average of $\operatorname{mf}_{\max }(\tau)$ between the ranges 300 and $350 \mathrm{~m}$. This was possible because the atmospheric conditions under which the experiment was carried out were relatively stable (evening); this is not a general procedure, and other 
methods should be employed to estimate the background noise level in other conditions.

\section{4) WAVEFORM DESIGN}

The ambiguity function is a design tool introduced by Woodward (1951) to help to understand the Doppler range ambiguity problem. Doppler range ambiguity arises when multiple frequencies are transmitted. Consider the case of a train of simple pulses where each subsequent pulse is a higher frequency. For a received reflection, there is now ambiguity between the estimated range and the Doppler frequency shift. In simple terms, a distant fast object and a closer slower object could be indistinguishable. The wideband ambiguity function $\Psi_{n}(t, v)$ is a two-dimensional function of time lag and radial velocity for a waveform $x(t)$ and a Doppler-shifted waveform $x_{d}(t)$ (Cahlander et al. 1964),

$$
\Psi_{n}(t, v)=\left|\sqrt{\alpha(v)} \int_{-\infty}^{\infty} x^{*}(t) x_{d}[\alpha(v)(\tau-t)] x d \tau\right| .
$$

A waveform with an ambiguity function that is a Dirac delta function at $t=0$ and $v=0$ has no range-velocity ambiguity. Thus, minimizing $\Psi_{n}(t, v)$, where $t \neq 0$ and $v \neq 0$, reduces the range-velocity ambiguity. Rangevelocity ambiguity manifests in a stepped chirp when the Doppler shift is equal to the frequency spacing: For example, if the first transmitted subpulse frequency matches the second frequency of the returned signal, then they are indistinguishable.

Doppler scales with frequency; therefore, the ambiguity is more problematic with higher frequencies. Hence, nonlinear frequency spacing makes best use of the available bandwidth. The experimental chirp sodar has a usable frequency range of $3-6 \mathrm{kHz}$. To optimize the available bandwidth, the transmitted frequencies was chosen using Eq. (13), where $f_{1}$ is the maximum operating frequency $(6 \mathrm{kHz})$ and each subsequent frequency $f_{n+1}$ is determined from the previous using the maximum expected radial velocity $V_{r \max }$ as follows:

$$
f_{n+1}=f_{n}-f_{n}\left(1-\frac{c-2 V_{r \max }}{c+2 V_{r \max }}\right) .
$$

The experimental sodar has a usable bandwidth of 3-6 kHz; assuming $V_{r \max }=7 \mathrm{~m} \mathrm{~s}^{-1}$, this means that a step chirp may have up to nine frequencies without ambiguity, and assuming $c=340 \mathrm{~m} \mathrm{~s}^{-1}$, this yields the following frequencies that were used: $6000,5525,5088$, 4686, 4315, 3974, 3660, 3370, and $3104 \mathrm{~Hz}$.

\section{5) NONCOHERENT DETECTION}

Because of the nature of the scattering volume, the relative phase of the subpulses in the returned signal is unpredictable. Therefore, coherent signal detection is not possible. However, a matched filter receiver can still be used to detect backscatter, but the lack of coherence will influence the performance. To investigate this, an approximate model of Bragg scattering was employed. The ambiguity function in Eq. (12) was evaluated for the step chirp, but the phase of each of the subpulses was randomized for the Doppler-shifted signal,

$$
\begin{aligned}
x_{d}(t, v)= & \sum_{m=1}^{M} \sin \left(2 \pi f_{m}\{\alpha(v)[t-(m-1) T]\}\right. \\
& \left.+\phi_{m}\right) G_{d}(t, v),
\end{aligned}
$$

where $\phi_{m}$ is a uniformly distributed random phase between $-\pi$ and $\pi$, generated independently for each subpulse. The ambiguity function was averaged from 40 repeated simulations for a nine-frequency stepped chirp with a subpulse length of $20 \mathrm{~m}$. This average represents the expected range-velocity ambiguity when a set of randomly located moving objects scatters the sound simultaneously but a different set of objects scatters each frequency. The ambiguity function for a single discrete moving object was also calculated; this is where the phase relationship is predictable and linear (coherent).

The left panel of Fig. 3 shows the marginalized velocity ambiguity function, and the right panel of Fig. 3 shows the marginalized range ambiguity for both coherent and noncoherent scattering. The levels of both are normalized to the maximum level for the coherent detector. The coherent detector offers an increased resolution and a 9-dB increase in signal level; this is a result of pulse compression. However, the noncoherent detection still shows a broad peak in the ambiguity function. By analyzing the return signal and locating this broad peak, a noncoherent matched filter can still be used to assess the range-velocity, but the increased resolution and range associated with pulse compression will not be realized.

\section{c. Analysis of sodar returns using GMMs}

While matched filter-based processing of sodar signals does not improve range or Doppler resolution, there are several other interesting advantages. It provides radial velocity profiles from multifrequency data without the need for separate processing for each frequency; conventional methods often use ad hoc methods for combining frequency information. Additionally, range gating is not required. This does not increase the resolution of the data but removes the need for smoothing or excessive overlapping if smoother data are required. It is also possible that reflections from fixed objects will maintain a 

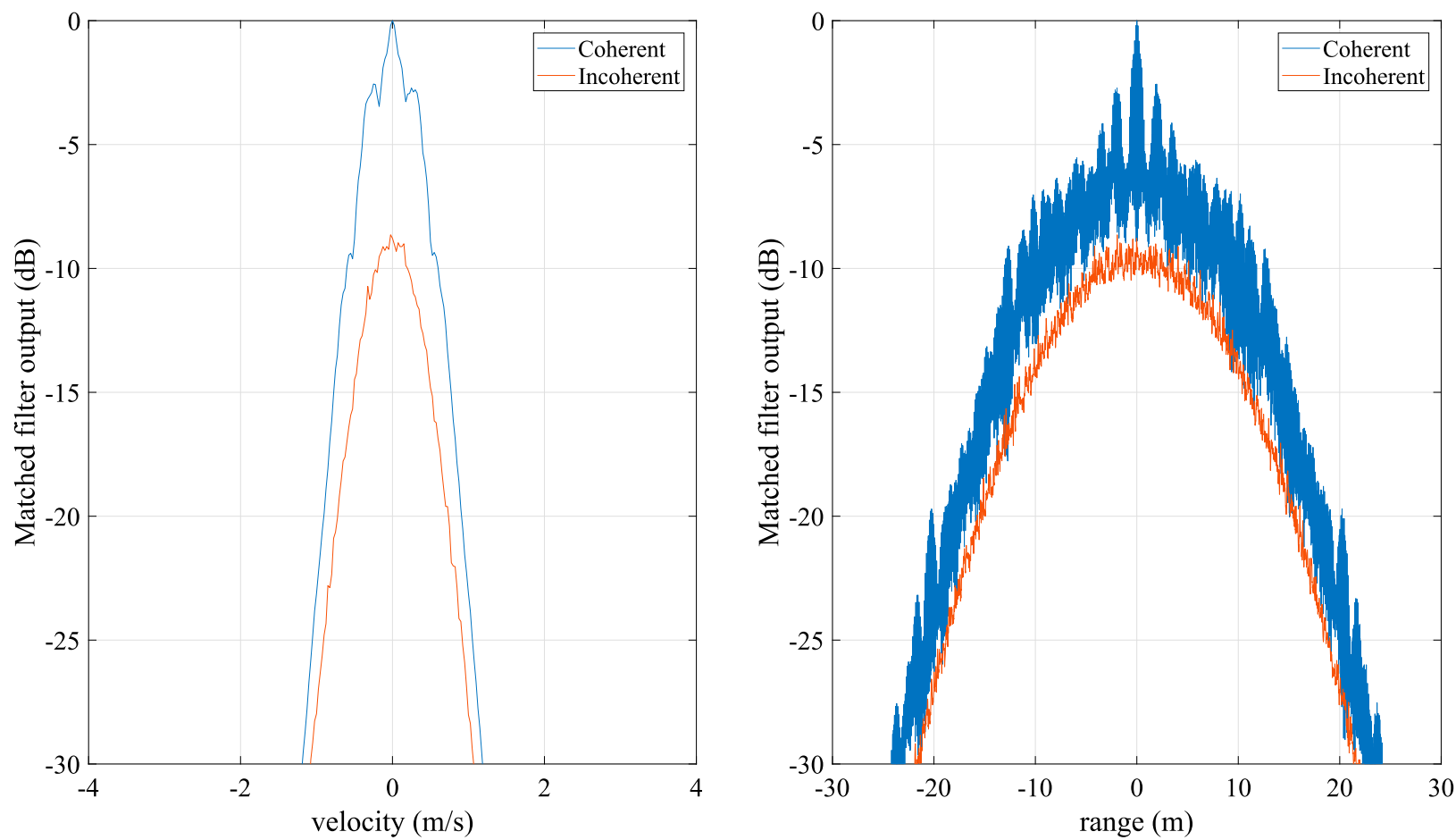

FIG. 3. Ambiguity function for a 180-m, nine-frequency stepped chirp, comparing the ambiguity function when phase response is linear (blue) and when phase response is random and unpredictable (red). Both ambiguity functions are normalized to the maximum level for the coherent method. Shown are (left) the marginalized velocity ambiguity and (right) the marginalized range ambiguity.

coherent subpulse phase relationship; this means that pulse compression may enable fixed echoes to be identified with increased resolution compared with backscatter.

Analysis of sodar signals has traditionally involved a statistical moment analysis of the peaks in spectral data. Within each range gate the mean and standard deviation of the radial velocity are computed, and postprocessing methods are used to reduce the influence of noise and fixed echoes on the radial velocity estimates. This usually involves rejecting data from range gates when either the SNR is too low or the spectrum is narrow. This reduces the data availability; it would be advantageous if the presence of fixed echoes did not mean rejection of data but could be robustly ignored. It is with this goal that Gaussian mixture models are applied to the matched filter result.

\section{BACKGROUND TO MIXTURE MODELS}

A mixture model is a probabilistic tool where it is assumed that the probability density function (pdf) of a random process can be approximated as a sum of other, simpler pdfs (e.g., Gaussian). Melnykov and Maitra (2010) trace back the history of statistical mixture modeling to Newcomb (1886) and Pearson (1895). The most popular form of mixture model is where the component mixtures are Gaussian distributions (GMM) (Day 1969; Wolfe 1970; McLachlan and Peel 1999; McLachlan and Peel 1999; Banfield and Raftery 1993). GMMs are used for cluster analysis, segmentation, and density estimation in many areas, including finance (Lindemann et al. 2004), audio signal processing (Reynolds and Rose 1995), image processing (Permuter et al. 2003), and medical applications (Schlattmann 2009) among many others.

Taylor's classical "frozen turbulence" hypothesis assumes that the atmosphere consists of many randomly located discrete scatterers. The velocity, range, and scattering strength of these objects is assumed to vary according to some underlying random process. By fitting a GMM to the matched filter output, the joint probability density function of this underlying processing can be estimated. From this, components that exhibit particular properties can be rejected. For instance, components with low variance in range and velocity are likely to be from fixed echoes.

The resulting GMM fit is a joint probability density function that represents the probability there is a 
scatterer at a particular range, radial velocity, and scattering strength. The joint probability density function $p_{x}\left(z, v_{r}, \mathrm{SNR}_{\text {matched }}\right)$ was modeled as a sum of Gaussian probability density functions,

$$
p_{x}\left(z, v_{r}, \mathrm{SNR}_{\text {matched }}\right)=\sum_{k=1}^{K} w_{k} N\left(x \mid \boldsymbol{\mu}_{k}, \boldsymbol{\Sigma}_{k}\right),
$$

where $x$ is the output from the matched filter, $N$ is a Gaussian probability density function, $k$ is the component number, $K$ is the total number of components, $w_{k}$ is the component weight, $\boldsymbol{\mu}_{k}$ is a three-dimensional vector of mean values for the $k$ th component, and $\boldsymbol{\Sigma}_{k}$ is a $3 \times 3$ covariance matrix for the $k$ th component. If $K$ is known, then the parameters of the GMM can be estimated using expectation-maximization (EM; Dempster 1977). Using conditional probability, the likelihood function is derived,

$$
\begin{aligned}
& \ln L\left(\boldsymbol{\mu}_{k}, \mathbf{\Sigma}_{k}, w_{k} \mid x_{1}, \ldots, x_{M}\right) \\
& \quad=\sum_{m=1}^{M} \ln \sum_{k=1}^{K} w_{k} N\left(x_{m}\left|x_{m}\right| \boldsymbol{\mu}_{k}, \mathbf{\Sigma}_{k}\right) .
\end{aligned}
$$

The maximum of Eq. (16) was found by optimizing the model parameters $\boldsymbol{\mu}_{k}, \boldsymbol{\Sigma}_{k}$, and $w_{k}$ over $M$ measurements. The EM algorithm is an iterative algorithm that searches for local maxima in the log-likelihood function to estimate the most likely set of model parameters. The number of components $K$ is chosen by computing the Akaike information criterion (AIC; Akaike 1974), which is a measure of goodness of fit for a model. The EM algorithm is carried out for a range of $K$, and the model with the lowest AIC is selected.

\section{d. Data capture and analysis}

The chirp sodar was operated at a site near Manchester, United Kingdom (Basell Polyolefins, Carrington, Manchester), at 2050 UTC 28 March 2012 for 10 min under stable atmospheric conditions. The sodar transponders were placed around $2 \mathrm{~m}$ apart on a flat concrete surface; the nearest object was a single-story building $60 \mathrm{~m}$ from the transponders. Additionally, a cooling tower, buildings, and a number of pipes were located between 100 and $200 \mathrm{~m}$ away. The presented data represent a limited time period and type of atmospheric condition; in future studies it will be important to understand how the method performs in a wider range of conditions, such as when convection occurs.

The device was operated using a nine-frequency stepped chirp. The length of each subpulse was $20 \mathrm{~m}$. Five beams were transmitted (respectively labeled 1-5): south, east, vertical, north, and west, using a tilt angle of $20^{\circ}$. The receiver captured $3.5 \mathrm{~s}$ of audio synchronized from the start of playback, and beamforming was carried out on the received signals. Radial velocities were estimated using both the noncoherent matched filter method and frequency estimation from power spectra.

For the power spectrum method, the return signal was windowed into range gates using $20-\mathrm{m}, 50 \%$ overlapping Hanning windows. A power spectrum was computed for each range gate, and a Gaussian function was fitted to the spectrum amplitude to estimate the frequency; this is a method commonly utilized in commercial sodar. The Gaussian fit was restricted to within $100 \mathrm{~Hz}$ of the $m$ th subpulse frequency and $(m-1) T / c$ seconds were removed from the start of the signal to account for the delayed transmission of each subpulse. Once the Doppler shift $d$ was estimated for each frequency and range, the radial velocity was computed using the following relationship:

$$
v=-\lambda d / 2 .
$$

The SNR of the backscatter was estimated as follows:

$$
\operatorname{SNR}_{\text {spec }}(z)=20 \log _{10}\left[\operatorname{Spec}_{\text {peak }}(z) / N_{\text {spec }}\right] \text {, }
$$

where $\operatorname{Spec}_{\text {peak }}(z)$ is the peak level of the amplitude spectrum in range gate $z$ and $N_{\text {spec }}$ is the noise level computed as the average peak spectrum amplitude from range gates between 300 and $350 \mathrm{~m}$. For each subpulse and range gate, the radial velocities were averaged over frequencies at which $\operatorname{SNR}_{\text {spec }}(z)$ was greater than $3 \mathrm{~dB}$; all range gates with insufficient SNR were rejected.

\section{Results and discussion}

\section{a. Noncoherent signal detection}

Figure 4 shows the matched filter radial velocity estimates from the westerly beam, overlaying $10 \mathrm{~min}$ of data (30 pulses). A matched filter bank resolution of $0.05 \mathrm{~m} \mathrm{~s}^{-1}$ was used. The $x$ axis shows the range, and the $y$ axis shows the radial velocity; the color indicates the matched filter output magnitude, or $\mathrm{SNR}_{\text {matched }}$. Figure 4 shows data points when $\mathrm{SNR}_{\text {matched }}$ was greater than $3 \mathrm{~dB}$.

Figure 4 has captured a radial wind velocity profile, showing an increase in radial velocity up to $70 \mathrm{~m}$ (the range of the device was generally limited by electronic noise). However, there were also several scatterers with radial velocities around $0 \mathrm{~m} \mathrm{~s}^{-1}$. These are fixed echoes resulting from several tall structures nearby. Fixed echoes were visible in the data up to $200 \mathrm{~m}$ away. 


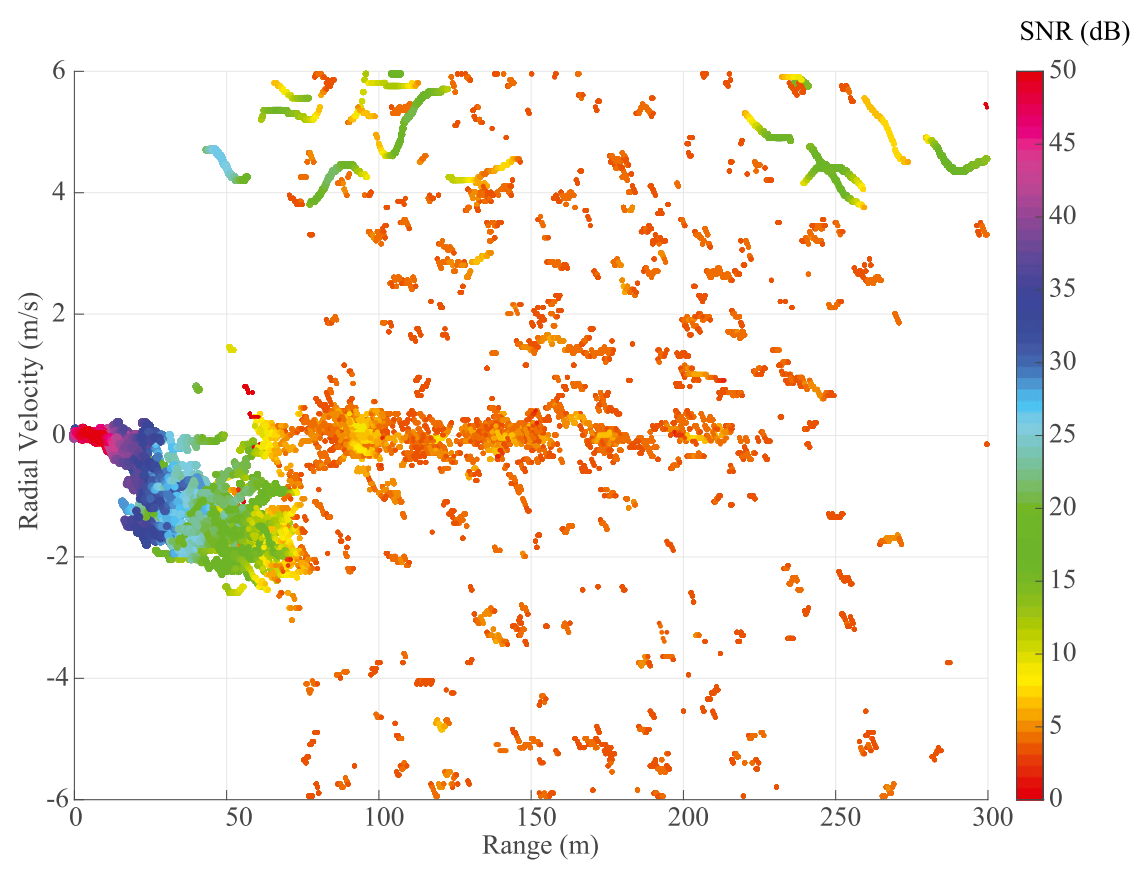

FIG. 4. Radial velocities estimated using matched filter estimates: the $x$ axis depicts the range, the $y$ axis represents the detected radial velocity, and colors represent the matched filter output magnitude (referenced to the average level between ranges of 300 and $350 \mathrm{~m}$ ). Each point represents the detected radial velocity for each range. The results from 30 stepped-chirp pulses are overlaid.

Figure 5 shows the radial velocity computed using the power spectrum method. As with the matched filter method, only data points that have an SNR that is $3 \mathrm{~dB}$ above the background noise level are plotted.

In comparing the data from the matched filter and spectral methods, it is seen that there is no difference in the maximum range where backscattered sound from turbulence can be detected (around $70 \mathrm{~m}$ ). If pulse compression were possible, there would be a clear advantage in the maximum range. This is consistent with Hargreaves et al. (2014); the matched filter is functioning only as a noncoherent detector.

Some fixed echoes visible in Fig. 4 are not apparent in Fig. 5. For example, echoes at a range of 175 and $200 \mathrm{~m}$ in the matched filter data cannot be seen in the spectral data. This is indicative of pulse compression. The returned sound is a reflection from a solid object, unlike turbulence, which exhibits backscattering. The reflected sound maintains the intersubpulse phase relationship; for fixed echoes, pulse compression appears successful.

In addition, there is a fixed echo at $60 \mathrm{~m}$, visible at $0 \mathrm{~ms}^{-1}$ in Fig. 4. In Fig. 5, the same fixed echo at $60 \mathrm{~m}$ is not clearly visible; however, it appears that the radial velocity estimates using the spectral method are lower. This is likely to be because of the spectral peak estimation; if there are two closely spaced peaks, the
Gaussian fit could be suboptimal and the radial velocity estimate could be biased.

\section{b. Analysis of sodar returns using GMM}

Figure 6 shows the result of the GMM fit to the data from Fig. 4. The AIC was evaluated for up to 60 components; the lowest AIC was when the GMM contained 50 components. To visualize the GMM, Fig. 6a shows each component plotted as an ellipse, where the location of the center is the mean and the surface represents 1 standard deviation $\sigma$. The color represents the strength of the backscattered signal $\mathrm{SNR}_{\text {matched }}$. The pdf for the GMM was plotted in Fig. 6b, where the shading represents the probability when the pdf was marginalized with respect to the matched filter output magnitude; this shows the probability of a moving scatterer at a particular range and radial velocity.

Figure 6a shows a number of components with very low velocity magnitude and velocity variance. Direct sound is visible up to around $20 \mathrm{~m}$. At $90 \mathrm{~m}$ there is a single small component with low variation in velocity and range, and from around 120 to $200 \mathrm{~m}$ there was a component with a large range variance but low velocity variance; these two components represent fixed echoes from nearby objects. The GMM pdf in Fig. 6b can be helpful in making sense of the response. As the GMM fit was computed over 30 pulses, it captures any 


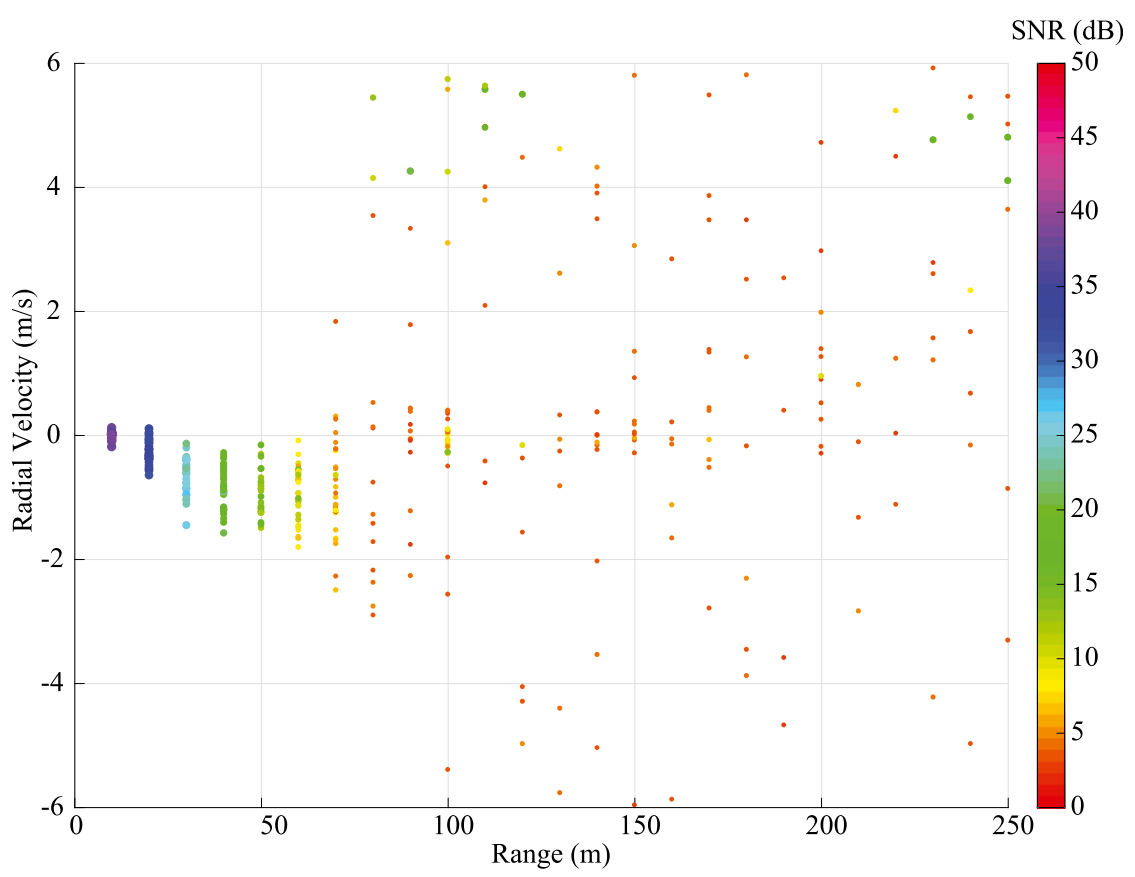

FIG. 5. Radial velocities estimated using spectral estimates: the data are windowed into range gates, the $x$ axis depicts the range, the $y$ axis represents the detected radial velocity, and colors represent the reflection strength (spectral peak level referenced to the average spectral peak level between ranges of 300 and $350 \mathrm{~m}$ ). Each point represents the detected radial velocity for a particular range gate. The results from 30 stepped-chirp pulses are overlaid.

consistencies in the response over the measurement period. Figure $6 \mathrm{~b}$ indicates that there is a high probability that a stationary object is located at around $90 \mathrm{~m}$. The use of the GMM pdf to analyze the response was further demonstrated by considering, in Fig. 4, the presence of scatterers between 50 and $100 \mathrm{~m}$ away with a radial velocity of around $5 \mathrm{~m} \mathrm{~s}^{-1}$. In Fig. $6 \mathrm{~b}$, this same region shows that the components have a relatively low probability; this is because the returns were not consistent across the 30 pulses and are likely just due to noise.

Some of the more distant components show a very high variance. These components were due to background (a)

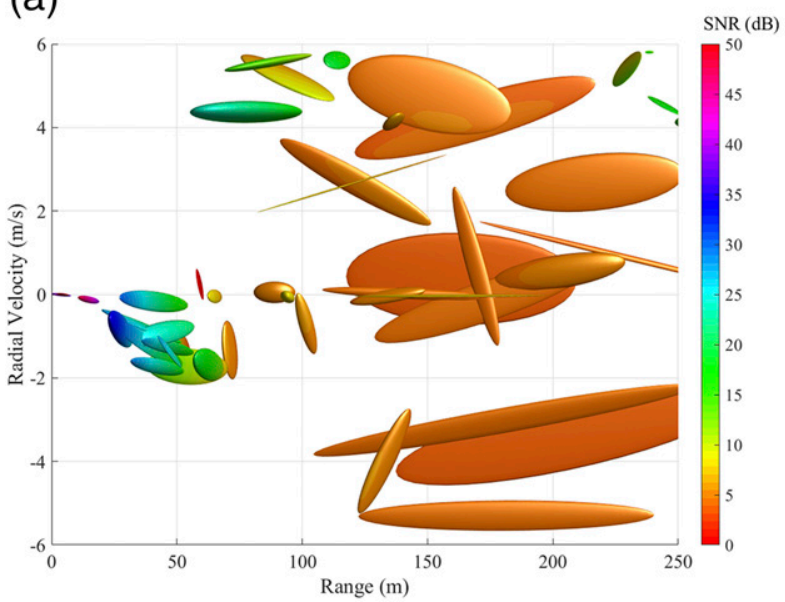

(b)

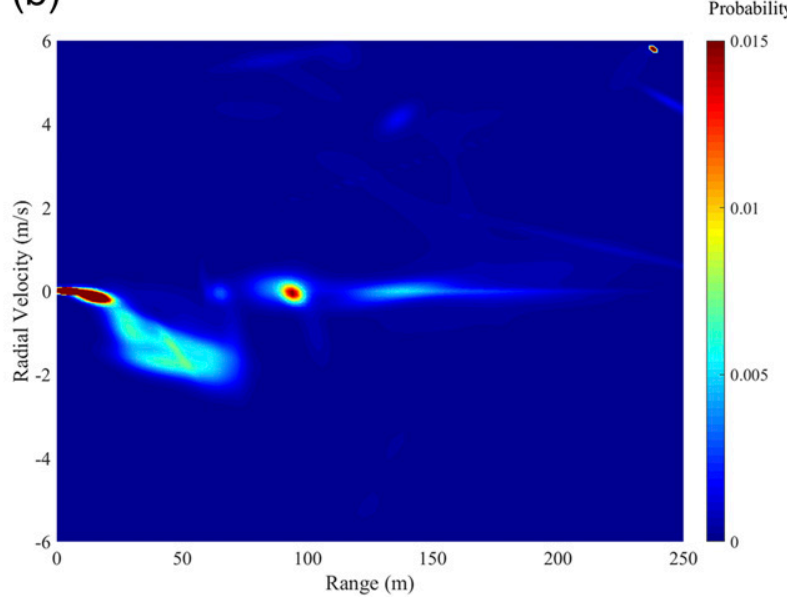

FIG. 6. (a) GMM fit to matched filter data from beam 5 (west): 50 components, the Gaussian components are displayed as ellipses, and colors represent magnitude of matched filter output. (b) Probability density function of GMM fit to matched filter data: 50 components, and colors represent the probability that reflection originated from a particular range at a radial velocity. 
(a)

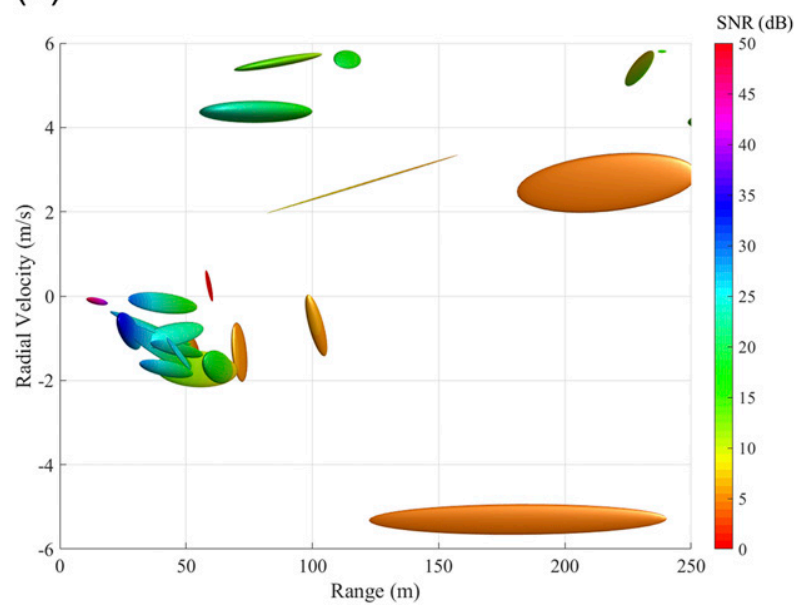

(b)

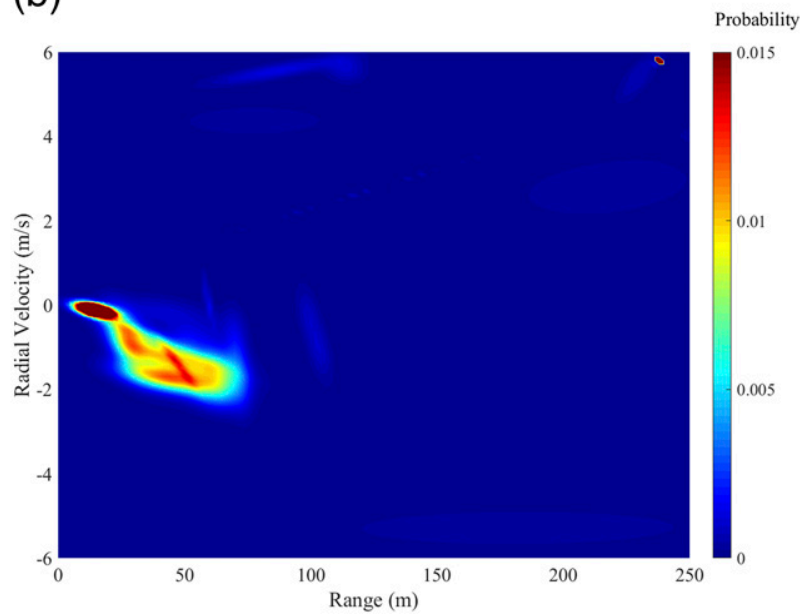

FIG. 7. (a) GMM fit to matched filter data from beam 5 (west), with noise and fixed echo components removed. (b) GMM pdf.

noise in the system. The matched filter was not detecting any strong returns; this is the matched filter output to a broadband random noise input. Additionally, many of the components from noise also do not decay with distance as one would expect the backscattered return to do; this fact could be used to identify noise.

\section{1) Cluster Analysis of SODAR Returns}

On the basis of the statistical parameters of each component $\left(\mu_{k}, \Sigma_{k}\right.$, and $\left.w_{k}\right)$ extracted from the GMM, rules were defined to classify each component as backscatter, fixed echo, or noise. Experience and guidance from Peters et al. (1984) were used to determine these rules. The classification rules were as follow:

- Components are classed as fixed echoes if the magnitude of the mean velocity $\mu_{v}$ is less than $0.1 \mathrm{~m} \mathrm{~s}^{-1}$ and the standard deviation of the radial velocity $\sigma_{v}$ is less than $0.1 \mathrm{~m} \mathrm{~s}^{-1}$. The rationale for this is that, while backscatter may have a low Doppler shift, it will have a much higher variance because of turbulence fluctuations.

- Components are classified as noise if the covariance matrix indicates that the matched filter output increases with range (scattering magnitude should decrease with range) and if $\sigma_{v}$ is greater than $1 \mathrm{~m} \mathrm{~s}^{-1}$.

After noise/fixed echo component classification, all remaining components were classified as backscatter. These rules were used to remove fixed echoes and noise (Fig. 7). This has removed direct sound, the strong reflection at $90 \mathrm{~m}$, the fixed echo between 120 and $200 \mathrm{~m}$, and most of the noise components.

The same process was carried out for each of the five beam directions. Figure 8 a shows the result of the GMM fit to the vertical beam (beam 3). Similar to beam 5, Fig. 8 b shows a high probability of scatter from objects with a low velocity magnitude and variance at $80-100$ and around $120 \mathrm{~m}$. It is important in the cluster analysis that the fixed echo rejection algorithm does not also reject backscatter. For backscatter with low radial velocity, this relies on the velocity variance being less than $0.1 \mathrm{~m} \mathrm{~s}^{-1}$. From Fig. 9, it can be seen that the fixed echoes at $80-100$ and around $120 \mathrm{~m}$ have been successfully rejected. A couple of components between 20 and $80 \mathrm{~m}$ have been rejected. Further refining of the procedure is likely required; for instance, a machine-learning approach to identification and rejection of fixed echoes may be more robust, although this was beyond the scope of this initial investigation.

\section{2) EXTRACTION OF WIND VELOCITY PARAMETERS}

The GMM pdf appears to be a useful tool for identifying fixed echoes, but it can also provide an estimate of the radial wind velocity profile. The matched filter results were analyzed: all data points with an SNR less than $6 \mathrm{~dB}$ were rejected. After the fixed echo components were removed, the GMM pdf was marginalized by summation over the matched filter output magnitude dimension. The 50th percentile (the median) of the radial velocity was computed for each range.

The standard deviation of the radial velocity is a commonly quoted meteorological parameter. Because the standard deviation requires a normal distribution and this is not, it can be approximated by averaging the distance from the median to the 159th and 841th permilliles, which for a normal distribution would represent 1 standard deviation around the mean.

To provide an indication of when the signal disappears below the noise floor, the cumulative distribution function is evaluated with respect to range. The range where 
(a)

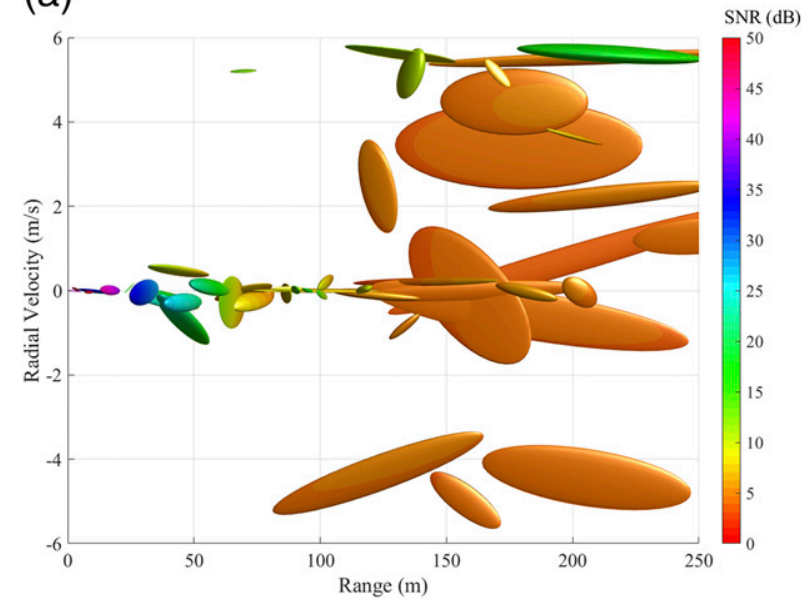

(b) Probability

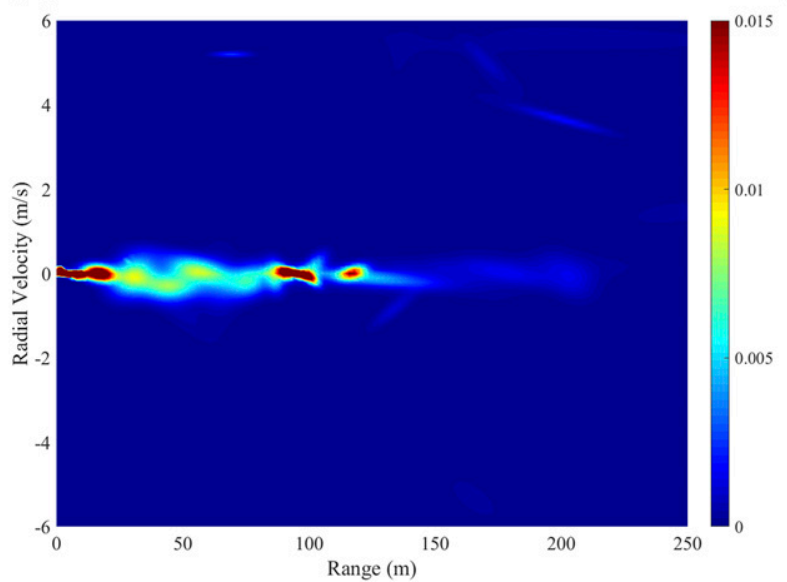

FIG. 8. As in Fig. 6, but fit to matched filter data from beam 3 (vertical).

this falls below 0.85 was chosen after empirical investigations. One of the idiosyncrasies with the EM algorithm is that, by randomly initializing the starting point, the GMM will be different for each fit. To eliminate the variability resulting from the stochastic nature of the fitting algorithm, the whole algorithm is repeated 30 times, and the resulting velocity magnitude and the velocity standard deviation profiles are averaged. Figure 10 shows the resulting radial velocity profiles for beams $1-5$, with dashed lines representing the standard deviation.

Sodar devices often assess data quality in the form of the percentage availability over a measurement period for a range gate (from $0 \%$ to $100 \%$ ). It may be possible to use the magnitude of the GMM pdf to indicate data quality; however, the collection and analysis would need to be carried out over a wider range of atmospheric conditions than how it is addressed in the current study to assess this.

\section{3) COMPARISON WITH SPECTRAL METHODS}

Radial velocity profiles for the five beams were computed using the power spectrum method. Data were rejected when the SNR is less than $6 \mathrm{~dB}$. Spectral peaks that were the same or close to the spectral width of the transmitted sound were likely to be from fixed echoes. The width of the spectral envelope of the transmitted peak was $7.7 \mathrm{~Hz}$ (the $\sigma$ of the Gaussian fitted to the spectrum). Data were rejected when the spectral peak width $(\sigma)$ was less than $9 \mathrm{~Hz}$; this threshold was determined empirically. Figure 11 shows the spectrally estimated radial velocity profiles. (a)

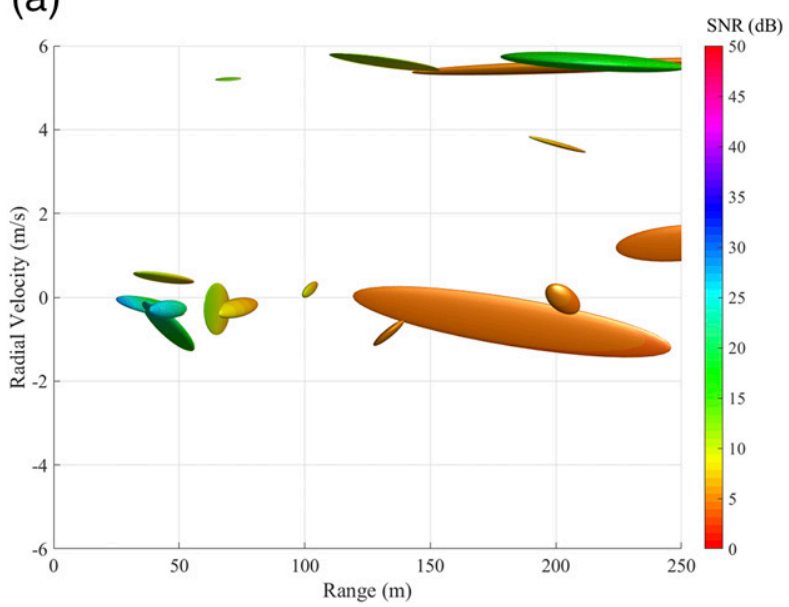

(b)

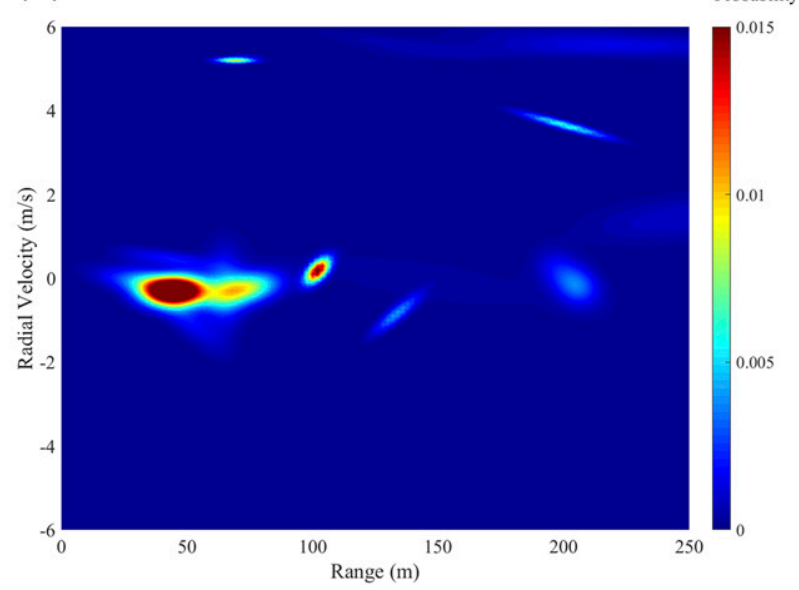

FIG. 9. As in Fig. 7, but fit to matched filter data from beam 3 (vertical). 


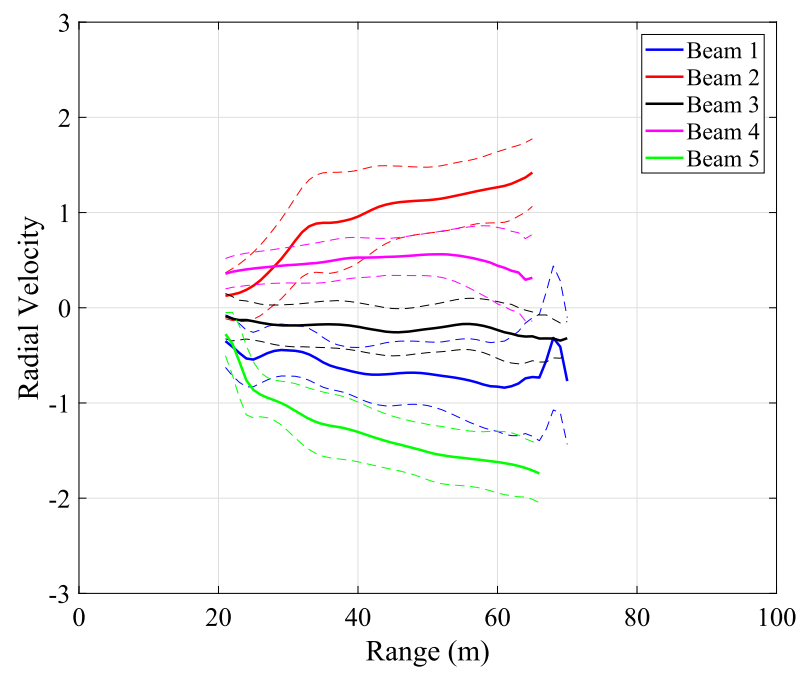

FIG. 10. GMM-detected radial wind velocity profile for all five beams; dashed lines represent 1 std dev.

In comparing the spectrum method with GMM methods (Figs. 10 and 11), it is seen that the maximum ranges are similar, around $70 \mathrm{~m}$, with the spectral method apparently offering a slightly farther range for some beams. However, the spectral method appears to consistently estimate a lower radial velocity magnitude compared with the GMM method. The reason for this is indicated by comparing beam 5 (west) and beam 2 (east) in Figs. 10 and 11. In the matched filter GMM results (Fig. 8), the east and west beams show similar wind velocity profiles but in opposite directions and at an equal distance from the vertical radial velocity. This was as expected; the device is capturing the same wind radial velocity field (east and west) but in opposite directions. However, in Fig. 11 beam 5 shows a sharp drop in the radial velocity at $65 \mathrm{~m}$ that is not replicated in beam 2 ; this is evidence that this profile was biased by fixed echoes from nearby objects. In general, the presence of clutter and nearby tall structures causes the spectrally estimated profiles to be underestimated. The similarity of radial velocity profiles in opposite directions indicates that the matched filter/GMM method is more robust to these fixed echoes. It is possible that sodar manufacturers have optimized their signal-processing algorithms to be more resilient to this problem, and therefore it would be important to validate this result on commercial systems.

The advantage provided by the GMM method comes from the greater number of dimensions in which the statistical analysis is performed. The GMM procedure captures how the range, radial velocity, and scattering magnitude all vary together, whereas the spectral analysis examines the radial velocity independently for each

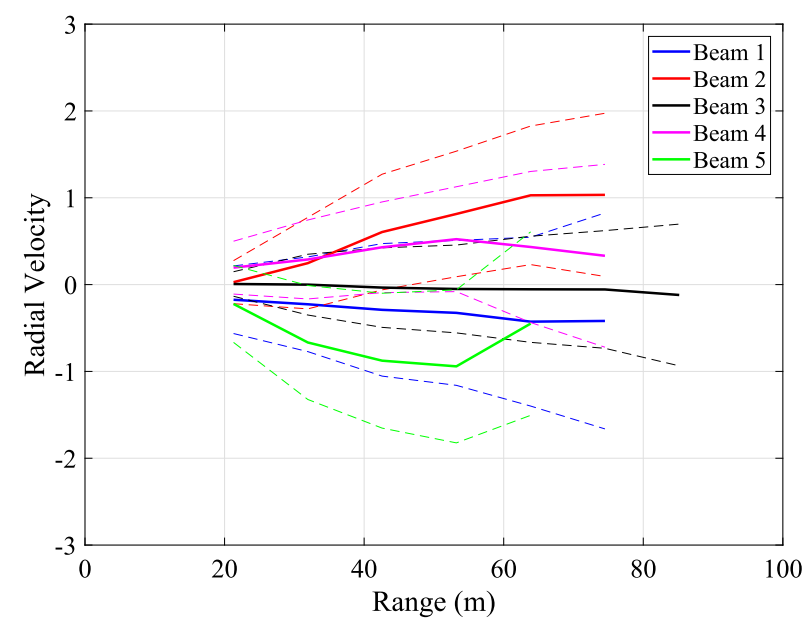

FIG. 11. As in Fig. 10, but for spectrally detected radial wind velocity.

range gate. The covariance captured by the GMM allowed the definition of a set of empirical clustering rules. In further developments performance may be improved by learning the best set of clustering rules experientially from simulations or in measurements with known fixed echo locations.

\section{Conclusions}

This paper presents a novel sodar analysis technique with some advantages in fixed echo detection and rejection compared with more traditional techniques. A flexible 64-channel bistatic sodar device was developed for which each transducer has its own signal path. This configuration enabled flexible beamforming, in which different frequencies can be steered electronically in the same direction; this is not possible on most commercial devices.

Backscattered return signals were analyzed using a noncoherent matched filter. This analysis method combined the estimation of Doppler shift from multiple pulses of different frequencies into a single optimization. Postprocessing of the matched filter data was carried out using Gaussian mixture modeling. This is a statistical analysis technique that utilizes the full dimensionality of the feature space to enable better identification and separation of unwanted sources from the backscattered signal. The proposed postprocessing method via GMM combines several aspects of sodar postprocessing previously carried out separately, in which profile estimation, fixed echo, and noise rejection are all combined into a single framework. In a limited study (over $10 \mathrm{~min}$ during stable atmospheric conditions), the processing method showed 
better performance in fixed echo detection and rejection over spectral methods, addressing one of the main limitations in sodar performance. However, careful siting of the instrument away from tall structures should still be the first consideration in the elimination of fixed echoes.

Acknowledgments. The authors acknowledge the U.K. Engineering and Physical Sciences Research Council for its support via Grant EP/G003734/1.

\section{REFERENCES}

Akaike, H., 1974: A new look at the statistical model identification. IEEE Trans. Autom. Control, 19, 716-723, https://doi.org/ 10.1109/TAC.1974.1100705.

Alberts, T. A., and P. B. Chilson, 2011: Evaluation of weather radar with pulse compression: Performance of a fuzzy logic tornado detection algorithm. J. Atmos. Oceanic Technol., 28, 390-400, https://doi.org/10.1175/2010JTECHA1409.1.

Antoniou, I., H. E. Jørgensen, F. Ormel, S. G. Bradley, S. von Hünerbein, S. Emeis, and G. Warmbier, 2003: On the theory of SODAR measurement techniques (final reporting on WP1, EU WISE project NNE5-2001-297). Ris $\varnothing$ National Laboratory Research Rep. Ris $\varnothing-\mathrm{R}-1410(\mathrm{EN})$, $59 \mathrm{pp}$.

AQSystem, 2013: AQ500 Windfinder user guide. Version 3, 171 pp. http://www.aqs.se/wordpress/wp-content/uploads/2013/12/ AQ500_UG_EN_131213_web.pdf.

Argentini, S., I. Petenko, A. Viola, G. Mastriantonio, I. Pietroni, G. Casasanta, E. Astridi, and C. Ghenton, 2013: The surface layer observed by a high-resolution sodar at DOME C, Antarctica. Ann. Geophys., 56, F0557, https://doi.org/10.4401/ ag-6347.

Banfield, J. D., and A. E. Raftery, 1993: Model-based Gaussian and non-Gaussian clustering. Biometrics, 49, 803-821, https:// doi.org/10.2307/2532201.

Bradley, S. G., 1999: Use of coded waveforms for SODAR systems. Meteor. Atmos. Phys., 71, 15-23, https://doi.org/ 10.1007/s007030050039.

__ and S. von Hünerbein, 2007: Comparisons of new technologies for wind profile measurements associated with wind energy applications. European Wind Energy Conference and Exhibition 2007, Vol. 3, WindEurope, 1747-1754.

,$- \ldots$, and T. Mikkelsen, 2012: A bistatic sodar for precision wind profiling in complex terrain. J. Atmos. Oceanic Technol., 29, 1052-1061, https://doi.org/10.1175/JTECH-D-1100035.1 .

Cahlander, D. A., J. J. G. McCue, and F. A. Webster, 1964: The determination of distance by echolocating bats. Nature, 201, 544-546, https://doi.org/10.1038/201544a0.

Day, N. E., 1969: Estimating the components of a mixture of normal distributions. Biometrika, 56, 463-474, https://doi.org/ 10.1093/biomet/56.3.463.

Dempster, A., 1977: Maximum likelihood from incomplete data via the EM algorithm. J. Roy. Stat. Soc., 39A, 1-38, http:// web.mit.edu/6.435/www/Dempster77.pdf.

Guimarães, D. A., and R. A. A. de Souza, 2015: Exploring practical aspects of the FSK modulation with non-coherent matched filter detection. Rev. Tecnol. Inf. Comun., 5, 22-28, https://doi.org/10.12721/2237-5112/rtic.v5n1p22-28.
Hargreaves, J. A., P. Kendrick, and S. von Hünerbein, 2014: Simulating acoustic scattering from atmospheric temperature fluctuations using a $k$-space method. J. Acoust. Soc. Amer., 135, 82-92, https://doi.org/10.1121/1.4835955.

Kalogiros, J. A., and C. G. Helmis, 1999: Automatic detection of fixed echoes in sodar facsimile records using the wavelet transform. J. Atmos. Oceanic Technol., 16, 734-738, https://doi.org/10.1175/1520-0426(1999)016<0734:ADOFEI> 2.0.CO;2.

Kendrick, P., S. von Hünerbein, and S. G. Bradley, 2010: A flexible new sodar design for chirped signals and conformable beam forming. Proc. 15th Int. Symp. for the Advancement of Boundary Layer Remote Sensing, Paris, France, ISARS.

Klauder, J. R., A. C. Price, S. Darlington, and W. J. Albersheim, 1960: The theory and design of chirp radars. Bell Syst. Tech. J., 39, 745-808, https://doi.org/10.1002/j.15387305.1960.tb03942.x.

Lindemann, A., C. L. Dunis, and P. Lisboa, 2004: Probability distributions, trading strategies and leverage: An application of Gaussian mixture models. J. Forecasting, 23, 559-585, https:// doi.org/10.1002/for.935.

Mayer, J., 2005: Characterisation of the atmospheric boundary layer in a complex terrain using SODAR-RASS. Diploma thesis, Dept. of Micrometeorology, University of Bayreuth, $86 \mathrm{pp}$, http://www.biomod.uni-bayreuth.de/mm/en/top/diss/ 29352Thesis_final.pdf.

McLachlan, G. J., and D. Peel, 1999: The EMMIX algorithm for the fitting of normal and t-components. J. Stat. Software, 4 (2), https://doi.org/10.18637/jss.v004.i02.

Melnykov, V., and R. Maitra, 2010: Finite mixture models and model-based clustering. Stat. Surv., 4, 80-116, https://doi.org/ 10.1214/09-SS053.

Newcomb, S., 1886: A generalized theory of the combination of observations so as to obtain the best result. Amer. J. Math., 8 , 343-366, https://doi.org/10.2307/2369392.

Othman, M. A. B., J. Belz, and F. Behrouz, 2017: Performance analysis of matched filter bank for detection of linear frequency modulated chirp signal. IEEE Trans. Aerosp. Electron. Syst., 53, 41-54, https://doi.org/10.1109/ TAES.2017.2649162.

Pearson, K., 1895: Contributions to the mathematical theory of evolution. II. Skew variation in homogeneous material. Proc. Roy. Soc. London, 186A, 343-414, https://doi.org/10.1098/ rsta.1895.0010.

Permuter, H., J. Francos, and I. H. Jermyn, 2003: Gaussian mixture models of texture and colour for image database retrieval. 2003 IEEE International Conference on Acoustics, Speech, and Signal Processing: Proceedings, Vol. 3, IEEE, 569-572, https:// doi.org/10.1109/icassp.2003.1199538.

Petenko, I., and Coauthors, 2014: Observations of optically active turbulence in the planetary boundary layer by sodar at the Concordia astronomical observatory, Dome C, Antarctica. Astron. Astrophys., 568, A44, https://doi.org/10.1051/0004-6361/ 201323299.

Peters, G., M. Latif, and W. J. Müller, 1984: Fluctuations of the vertical wind as measured by Doppler-SODAR. Meteor. Rundsch., 37, 16-19, http://eprints.uni-kiel.de/ 13252/1/Meteorol.\%20Rdsch. \%2037\%2C\%2016-19\%20\% 28Februar\%201984\%29.pdf.

Quintarelli, F., and A. Bergstrom, 2001: Numerical modeling of the spectral broadening of sodar echoes by winds perpendicular to the axis of a finite beamwidth antenna. 
J. Acoust. Soc. Amer., 109, 116-121, https://doi.org/10.1121/ 1.1331677.

Rao, I. S., V. K. Anandan, and M. S. Kumar, 2009: Multifrequency decoding of a phased array Doppler sodar. J. Atmos. Oceanic Technol., 26, 759-768, https://doi.org/10.1175/ 2008JTECHA1166.1.

Reynolds, D. A., and R. C. Rose, 1995: Robust text-independent speaker identification using Gaussian mixture speaker models. IEEE Trans. Speech Audio Process., 3, 72-83, https://doi.org/10.1109/89.365379.
Schlattmann, P., 2009: Medical Applications of Finite Mixture Models. Statistics for Biology and Health, Springer, 246 pp., https://doi.org/10.1007/978-3-540-68651-4.

Wolfe, J. H., 1970: Pattern clustering by multivariate mixture analysis. Multivar. Behav. Res., 5, 329-350, https://doi.org/ 10.1207/s15327906mbr0503_6.

Woodward, P., 1951: Information theory and the design of radar receivers. Proc. IRE, 39, 1521-1524, https://doi.org/10.1109/ JRPROC.1951.273638. 